\title{
A New 6 DOF Robotic Arm with Linkage Motion Mechanism and Actuators Placed in Base
}

\author{
Mohsen Shahhosseini, Rambod Rastegari, Roozbeh Abbasi \\ Department of Mechanical Engineering, School of Engineering, Islamic Azad University, Parand branch, Parand, Iran
}

\begin{tabular}{l}
\hline \hline Article Info \\
\hline Article history: \\
Received Nov 18, 2015 \\
Revised Jan 28, 2016 \\
Accepted Feb 22, 2016 \\
\hline
\end{tabular}

\section{Keyword:}

Kinematic Simulation

Linkage Mechanism

Parallel Mechanism

Related Actuators

Robotic Arms

\begin{abstract}
We examined mechanism design and kinematic simulation of a new six degrees of freedom (DOF) robotic arm with rotational joints and a linkage motion mechanism. In the design, a parallel linkage mechanism, accompanied by an additional set of bevel gears, was used to create the desired motion for all six links along with transfer of all actuators to the robot's base to reduce the mass of most of the arms. These changes resulted in reduction of the torque required for joints 1,2 , and 3 . Using this parallel mechanism ensures dependence to motion links and creates a special case for the control of the robot and more rigidity against unwanted movement. Initially, we examined mechanism design methods for a parallel linkage mechanism and considered methods for application in an operational robot. In the next step, we determined the kinematic relationships that were established between the robot's actuators and joints spaces due to the use of this mechanism. Then, we developed an example of the robot's function in a performance simulation. The simulation results indicated that the mechanism and controller performance were acceptable.
\end{abstract}

Copyright (C) 2016 Institute of Advanced Engineering and Science. All rights reserved.

\section{Corresponding Author:}

Mohsen Shahhosseini,

Department of Mechanical Engineering, School of Engineering,

Islamic Azad University, Parand branch,

Shahid Bahonar Blvd, Parand, Iran.

Email: mohsen.sh.hosseini@gmail.com

\section{INTRODUCTION}

The robots used in most industries are $6 \mathrm{DOF}$ robotic arms [1], because they have the ability to position and rotate for their end effectors with high accuracy. In the past few decades, various mechanisms have been considered to provide 6 degrees of freedom for the motion of robotic arms, with the robotic arms with rotational joints and wrists with intersecting axes being used most often. Currently, most industries use such robots, and Figure 1 shows the usual mechanism of motion for these robots. Due to the intersecting axes of the robot's wrist, the entrance rotational axes for joints 4,5 , and 6 must be set up by a rectifier mechanism, such that it is placed in arm 3. Therefore, the motors that move these three joints must be placed beside each other at the beginning of arm 3 (Figure 1, No. 8). The motors of the other three joints (joints 1, 2, and 3) are attached directly to the rotational part of the joints.

Now, it is obvious that, in the usual mechanism of such robots, the motors of joints 1,2 and 3 must move motors of next joints as well as the links after them. Therefore, moving the motors of these joints requires more torque than was required to move just the structure of the links of the robot. In previous years, different mechanisms have been suggested to improve the performance of such robots systems by decreasing the power required by their motors. Some of these mechanisms that have been used are the cable mechanism $[2,3,4]$, and the belt mechanism [5, 6, 7], which have similar functions, as well as hydraulic [8, 9, 10], pneumatic $[11,12,13]$, and linkage $[14,15,16]$, mechanisms. These mechanisms can cause motions to occur by transferring the robots' motors (such as cable, belt, and linkage mechanisms) or their main source of 
producing power (such as hydraulic and pneumatic mechanisms) to the robot's base, thereby transferring motion from these motors to the rotational axes of the related joints.

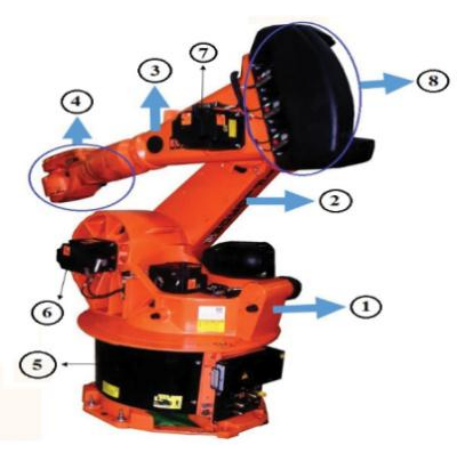

Figure 1. Usual mechanism for motion structure of 6 DOF Robotic arms: 1- Link 1; 2- Link 2; 3- Link 3; 4Robot wrist including links 4, 5, and 6; 5- place of setup motor for joint 1; 6- motor of joint 2; 7- motor of joint 3; 8- motors of joints 4, 5, and 6. (The picture belongs to Robotic arm, product of KUKA Company)

In this article, we describe our successful mechanism design and kinematic simulation to create six degrees of freedom for motion in a robotic arm, which allowed us to effectively control its motion. The functionality of this system is based on parallel mechanisms [17, 18]. In this mechanism we have used specially from establishment of some 4-bar linkage mechanisms in a parallel combination. By so doing, we were able to transmit the rotational motion of the motors of joints 3, 4, 5, and 6 (all of which were placed in the Robot's base and in the link 1) to its own main rotational axis for joint 3 and to the rectifier mechanism for the other joints. We should notice to this issue that the high rigidity of parallel robots (and parallel mechanisms) is one of the characteristics for which they are selected for some applications [18]. The main reason for selection of a parallel linkage mechanism by us, was to assist the robot in being rigid against unwanted movement, which is very important in terms of robot's vibration and control of its motion. In fact, the location and performance of the motors of joints 1 and 2 were the same as they are in the usual mechanisms for such robots, since changing the position of the motor of joint 1 is impossible, and, in joint 2 , tangible change in the amount of imported torque on the motor of joint 1 is ineffective.

In section two of this article, we show the design method of this motion transfer mechanism, we designed a CAD model for an operational 6 DOF robotic arm based on this mechanism, and at last we provide the kinematic relationships between the actuators space and the joints space of the robot with this mechanism. In third section, an open-loop control method used in kinematic simulation was reviewed. Moreover, the kinematic specifications of the simulated robot, simulation payload, and finally, the design of a simulation path and implementation approach, were done in MATLAB SimMechanics software were reviewed. In the section four, we provide the results of kinematic simulation of the robotic arm's function in MATLAB SimMechanics software and various graphs showing the results of the simulation, and in the last section we will express the conclusions obtained from the simulation results.

\section{MECHANISM DESIGN OF MOTION TRANSFER SYSTEM}

The basis of the functionality of the mechanism presented here was established on the parallel combination of some of 4-bar linkage mechanisms. The process of transferring rotational motion by the 4-bar linkage mechanism (C-C) [Crank - Crank] is illustrated in Figure 2.

In Figure 2, we can see transmission of rotary motion from the actuator motor (Point O1) to the follower link's center of rotation (Point O2). Further, the D link is rotated by the motor, because the parallelogram geometric model of the 4-bar linkage (C-C) mechanism and the F link will turn according to its center of rotation. So the $\mathrm{D}$ and $\mathrm{F}$ links always will be parallel.

Figure 3 shows the efficacy and interaction of the rotational motion of an independent joint that's concentric with the rotational center of the driver link of 4 bar linkage mechanism based on the procedure of rotational motion of that 4-bar linkage mechanism, which is placed in the internal space of linked arm. It should be noted that the design and use of an appropriate bearing installation will make the rotational movement of the 4-bar linkage mechanism's driver link independent of the rotation of the joint's axis. 

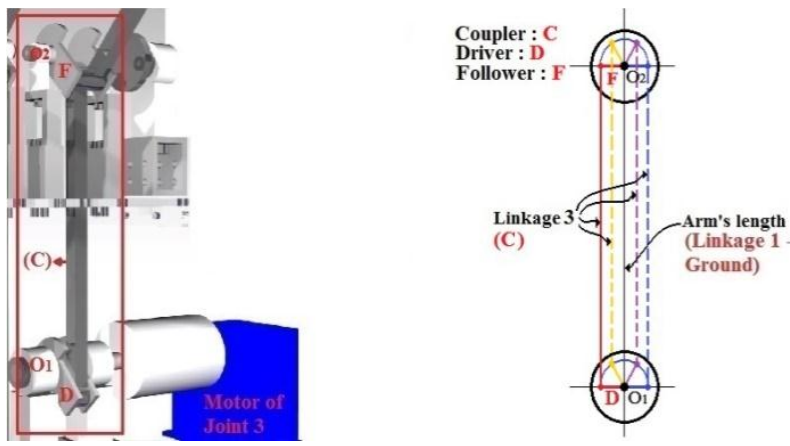

Figure 2. Right: Transition procedure of rotational motion by the 4-bar linkage mechanism (Crank - Crank). Left: An equivalent functional structure with this mechanism was also provided on the right side, at the base of the robot's motion mechanism. It's a linkage mechanism of joint 3 and from the motor of that joint up to the joint 2 axis.

According to Figure 3, we determined that by rotating joint and the attached arm to $\alpha$ degrees, assuming that the driver link of 4 bar linkage mechanism (D) is fixed in the arm of this construction, because of the parallelogram structure of 4 bar linkage mechanism, the coupler link (C) will rotate $\alpha$ degrees with respect to its original state. This means that there will be no rotation for the follower link $(\mathrm{F})$, and it remains fixed to its original state. According to the conditions described above, links D and F, will always remain parallel to each other irrespective of the amount of rotation of the arm's joint that contains the 4-bar linkage mechanism.

Thus, the procedure of interaction and the effect of rotational motion of a joint on the performance of the linkage mechanism make it possible to transfer rotational motion to the next joint or to the rectifier mechanism. Figure 4 shows an example of this in the connection between joints 2 and 3 . In the figure (right), two aligned arms are visible in three different conditions in which we can define the orientation of link No. 3 concerning the rotation of joint No. 2 and by the rotation of the motor of joint 3 .
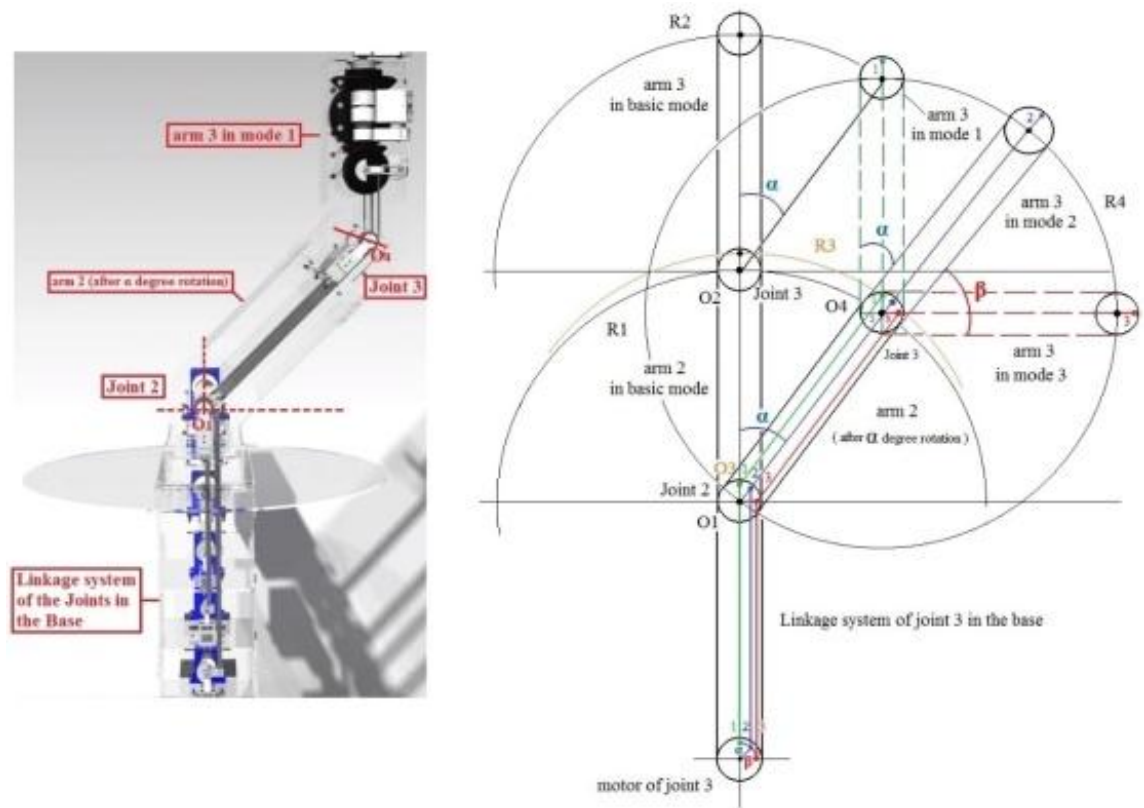

Figure 3. Right: Procedure of influencing the rotation of joint 2 on the performance of the motion transfer linkage mechanism of joint 3 (and similarly for joints 4, 5, and 6): Mode 1 is the initial mode of the motor of joint 3 (green); Mode 2 is the motor of joint 3 after rotating $+\alpha$ degrees (blue); Mode 3 is the motor of joint 3 after rotating $+(\alpha+\beta)$ degrees (red). Left: An equivalent functional structure with this mechanism was also provided on the right side, the mechanism was applied from the base up to arm (Link) No. 3 of the robot's motion mechanism. 
In Figure 4, generally, we want to consider the relationship between the motion of the said mechanism and the rotation of the joints that are placed in the path of mechanism movement. This Figure shows that the linkage mechanism of joint 3 from the motor of that's joint to joint 2 has the same conditions as in Figure 2. Also, the next part of the linkage mechanism inside link 2 and from joint 2 to joint 3 has the same conditions as in Figure 3. Hence, according to what we learned about the 4-bar linkage mechanism in Figures 2 and 3, it is apparent that the final follower link and the initial driver link always are parallel to each other. Since the final follower link is the driver for joint 3 in this figure and determines how the attached arm should be located, arm 3 always will remain parallel to the first driver link, and it is connected to motor of joint 3 in the robot's base. Considering the three conditions shown in Figure 4, we have the following:

The first condition: The motor of joint 3 is in the initial state and is not rotating; joint 2 has $+\alpha$ degrees rotation (assuming that the direction of normal vector of rotation is inward the plate). Now, we can see that the arm of joint 3 has deviated $-\alpha$ degrees from link No. 2, therefore we have:

$$
0^{\circ}-\left(+\alpha^{\circ}\right)=-\alpha^{\circ} \rightarrow \theta_{3 m}-\theta_{2 a}=\theta_{3 a} \rightarrow \theta_{3 a}+\theta_{2 a}=\theta_{3 m}
$$

* m: rotation related to the motor of joint; a: rotation related to the joint.

The second condition: The motor of joint 3 has been rotated $+\alpha$ degrees, and joint 2 also has $+\alpha$ degrees of rotation. Now, we can see that links No. 3 and No. 2 are in the same direction. Therefore we have:

$$
\left(+\alpha^{\circ}\right)-\left(+\alpha^{\circ}\right)=0^{\circ} \rightarrow \theta_{3 m}-\theta_{2 a}=\theta_{3 a} \rightarrow \theta_{3 a}+\theta_{2 a}=\theta_{3 m}
$$

The third condition: The motor of joint 3 is rotated $+(\alpha+\beta)$ degrees, and joint 2 is rotated $+\alpha$ degrees. Now, we can see that the arm of joint 3 has deviated $+\beta$ degrees from the arm of joint 2 . Therefore we have:

$$
\left(+\alpha^{\circ}+\beta^{\circ}\right)-\left(+\alpha^{\circ}\right)=+\beta^{\circ} \rightarrow \theta_{3 m}-\theta_{2 a}=\theta_{3 a} \rightarrow \theta_{3 a}+\theta_{2 a}=\theta_{3 n}
$$

Now, we have discussed the conditions of the relationships of motion between the rotation of joint 3 , rotation of joint 2, and rotation of the motor of joint 3 in all three conditions. After calculating the equation creating these conditions, if we want to distribute this relationship to linkage mechanisms of joints No. 4, 5 and 6 , because the linkage mechanisms of these joints from the base of the robot up to the joint 3 are similar to linkage mechanism of joint 3 , for all of the linkage mechanisms of these four joints $(3,4,5$ and 6$)$, we have a relationship between the motion of linkage mechanisms and the rotations of joints No. 2 and 3 .

$$
\theta_{3 a}+\theta_{2 a}
$$

This equation (No. 4) is equal to $\theta_{3 m}$ (required rotation for motor of joint 3) for joint 3's rotation procedure, and equal to the affected angle on the linkage motion mechanisms of joints No. 4, 5 and 6 from rotations of joints No. 2 and 3. We must bear in mind that the rotation of joint No. 1 has no influence on the motion of the connectors of the 4-bar linkage mechanisms.

Up to this step, we have discussed how the rotation of joints 1, 2, and 3 occurs, and, also, the dependency of the motion of joint 3 to joint 2 was explained. For other links $(4,5$, and 6$)$, we transfer rotational motion by means of a linkage mechanism from their motors to their connection points to the set of bevel gears. From this step onwards, we have a mechanism that is approximately similar to the usual mechanism of these Robots, i.e., a rectifier mechanism with three couples of bevel gears where the ratios between them are pairwise equal to 1 and all are formed at $90^{\circ}$ angles. This mechanism changes the rotational axes of the motion transfer system of joints 4, 5, and 6 from the related linkage mechanism on a 3-uniaxial, nested shafts. Figure 5 shows the structure of this mechanism as a CAD model. 


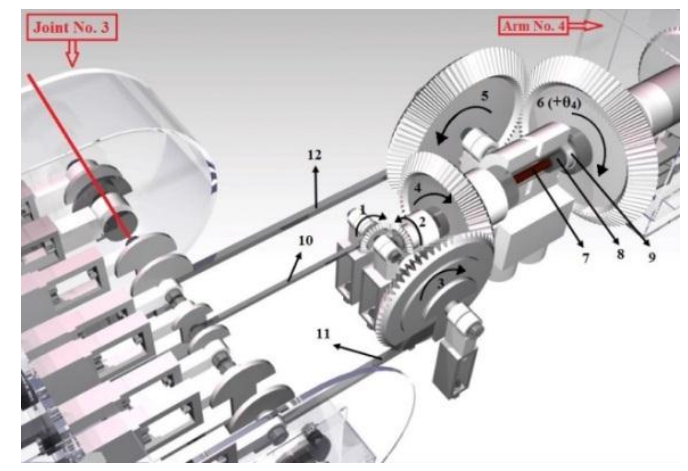

Figure 5. Structure of the rectifier mechanism for motion transfer of joints 4, 5, and 6, which lies in arm 3: 1) motive gear of joint 6 connected to motion transfer link; 2) follower gear of joint 6 connected to motion transfer shaft; 3) motive gear of joint 5 connected to motion transfer link; 4) follower gear of joint 5 connected to the motion transfer shaft; 5) motive gear of joint 4 connected to the motion transfer link; 6) follower gear of joint 4 connected to the motion transfer shaft; 7) motion transfer shaft of joint 6 ; 8) motion transfer shaft of joint 5 ; 9) motion transfer shaft of joint 4 ; 10) related motion transfer link of linkage mechanism of joint 6 ; 11) related motion transfer link of linkage mechanism of joint 5 ; 12) related motion transfer link of linkage mechanism of joint 4. (The rotational directions drawn in this figure are to create rotation in the positive direction for joints 4,5 , and 6.$)$

After this step, the motion transfer shaft related to joint 4 from the rectifier mechanism is connected directly to the link's arm and to the motion transfer shafts for rotational motion of joints 5 and 6 by means of three couples of bevel gears where the ratios between them are pairwise equal to 1 and all are formed at $90^{\circ}$ angles. These bevel gears form the structure of the robot's wrist and rotate the joints No. 5 and 6 axes [19]. Figure 6 [19] shows a view of the structure of the components of this part (Robot's wrist) as a CAD model.

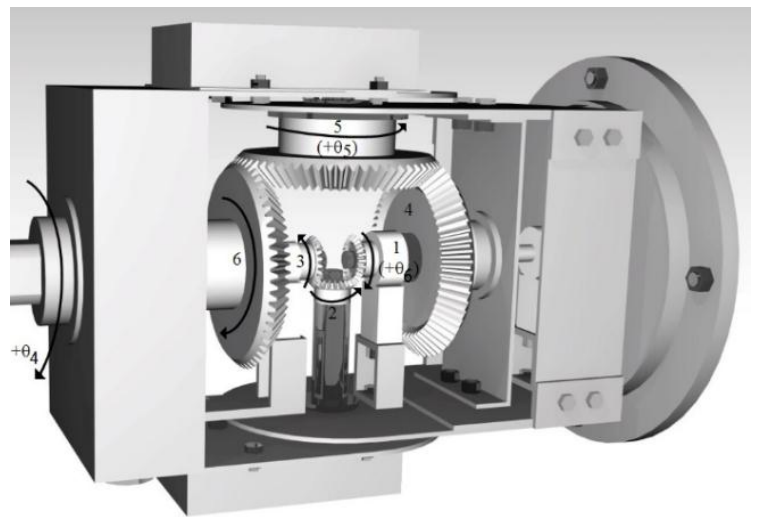

Figure 6 [19]. Structure of Robot's wrist mechanism to impose desired motion of joints 5 and 6: 1) motive gear connected to the main shaft of joint $6 ; 2$ ) middle gear and waste rotation of joint $6 ; 3$ ) gear connected to motion transfer shaft of joint 6; 4) fixed gear without any rotation for load support of gear No. 5; 5) motive gear connected to main shaft of joint $5 ; 6$ ) gear connected to motion transfer shaft of joint 5 . (The circular sides illustrated in this figure are to create rotation in the positive direction for joints 5 and 6. .)

Regarding this point, it is essential to know that there are interactions and dependency between the motion components in the wrist and the rectifier mechanisms of the Robot, i.e. the gears of joints 4, 5 , and 6 . This case is very important to compute the kinematic control or simulation of Robot. This issue is discussed in section 2.2. The widespread use of bevel gears for motion transfer in this mechanism, and since there is always backlash when using gears, and actually, this issue has a direct effect on a robot's dynamic control procedure. It's a very important issue that we must use from zero-backlash gearbox systems and sets in the manufacturing process of the commercial and operational robots with this mechanism. Utilizing this approach allows for improve dynamic control of the robot and performance accuracy to an acceptable level.

\subsection{Designing a CAD Model of an Operational 6 DOF Robotic Arm}

A New 6 DOF Robotic Arm with Linkage Motion Mechanism and Actuators ... (Mohsen Shahhosseini) 
Based on the discussion of the linkage mechanism and its use to create 6 degrees of freedom in the motion of robotic arms with rotational joints, we attempted to design a CAD model of a 6 DOF robotic arm with rotational joints and a wrist with intersecting axes. Figs. 7, 8 and 9 provide pictures of the CAD model for this robotic arm and associated information about the main parts that form it. For this purpose, this robot was checked by means of CATIA DMU Kinematic software for considering this fact that none of internal or external components should conflict with each other. We have used data from the textbook entitled "Tabellenbuch Metall" (reference No. [20]), to choose models and specifications and to calculate mechanical part sizes (for example: screws, nuts, bearings and etc.). These specifications were used in the design of the CAD model for this operational robotic arm. Robot's component materials include: (1) steel 304, (2) aluminum (Al 2219- T87) and (3) titanium (Tit / Ti-8Al-1Mo-1V) and their mechanical properties are presented respectively in reference No. [21].

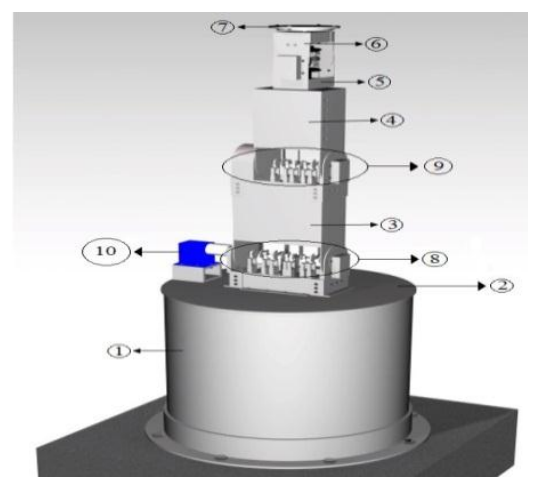

Figure 7. Overall view of the outer structure of the CAD model of the 6 DOF Robotic arm: 1) Robot's base (location of motors of joints); 2) link No. 1; 3) link No. 2; 4) link No. 3; 5) link No. 4; 6) link No. 5; 7) link No. 6 (end effector); 8) joint 2; 9) joint $3 ; 10$ ) motor of joint 2.

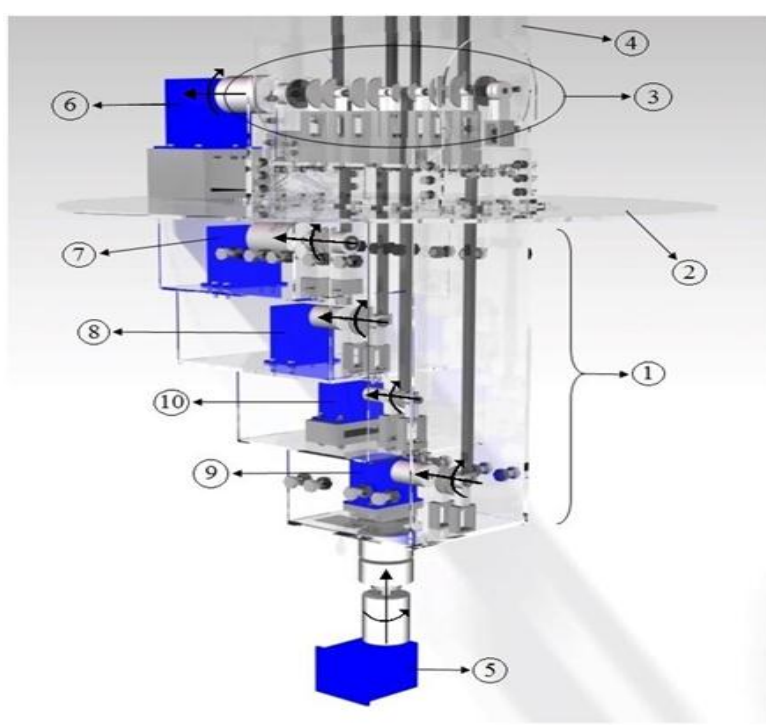

Figure 8. view of the inner structure of the robot's base on which the motors for the joints are placed: 1) container of motors and the links connected to them in robot base; 2) link No. 1 (arm of joint 1$)$; 3) joint $2 ; 4)$ link No. 2; 5) motor of joint $1 ; 6$ ) motor of joint 2 ; 7) motor of joint 3 ; 8) motor of joint 4 ; 9) motor of joint 5 ; 10) motor of joint 6 .

\subsection{Kinematic Relationships between Joints Space and Actuators Space}

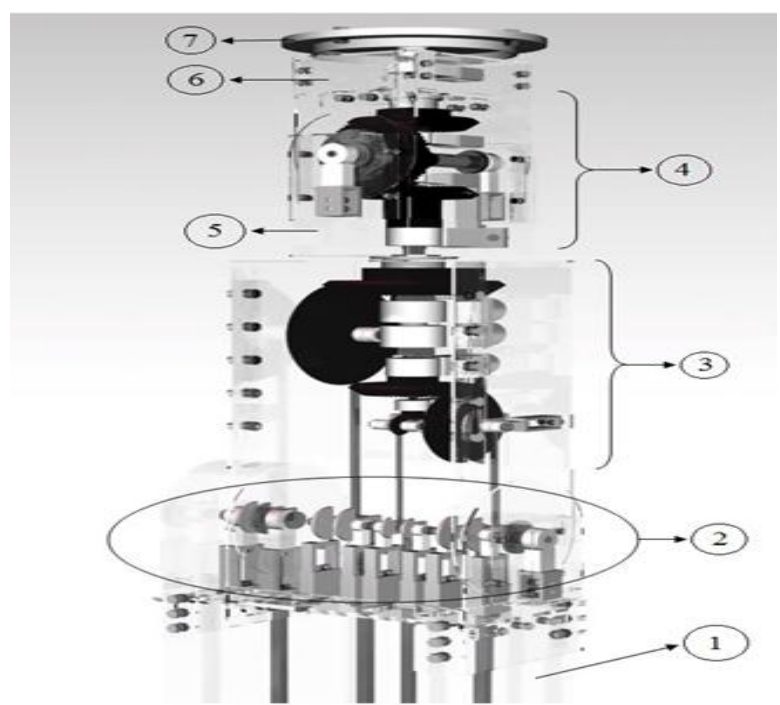

Figure 9. a view of the inner structure of link No. 2 ,until the end effector of robot where the arrangement of motive components of mechanism is observed: 1) arm of joint No. 2 and the links in it; 2) joint 3 ; 3) link No. 3 and set of gears of rectifier mechanism in it; 4) set of gears of mechanism of robot wrist; 5) link No. 4; 6) link No. 5; 7) link No. 6 (end effector) 
As mentioned in the Introduction, the rotations of the joints in this robot are interrelated, and the rotational motions of joints 3 through 6 are dependent on the rotations of the other joints. It is very important to consider this issue in the kinematic simulation of the robot. In fact, without knowing this relationship, it is not possible to control or simulate a robot with such a motion transmission mechanism. To do this and to calculate the relationship between the joints' space and the actuators' space, we reviewed and calculate the latest mobile robot parts, i.e., the end effector to its base and the motors of its joints. According to Figure 6, which shows the structure of the robot's wrist and method of rotation of joints 5 and 6 . The rotational angles of gears No. 1 to No. 6, which are the only effective parts in this section and ratios between them are pairwise equal to 1 , are equal to: for gears No. $1:+\theta_{6}$, No. $2:+\theta_{6}$, No. $3:+\theta_{6}+\theta_{5}-\theta_{4}$, No. $4: 0^{\circ}$, No. $5:+\theta_{5}$ and No. 6: $+\theta_{4}+\theta_{5}$. These amounts are given based on the practical rotation angle of the robot's main joints. And also for the rotation of joints 5 and 6 in the positive direction.

In the following and according to the values derived from Figure 6, we review Figure 5 and the performance of the rectifier mechanism. It is important to note in this section that the rotational motion of gears No. 2 and 4 in Figure 5 is equal to the amount of rotation of gears No. 3 and 6 in Figure 6, because the gears are joined mutually to each other by means of nested shafts 7 and 8, as shown in Figure 5. Considering this fact, we examined the rotational angles of gears No. 1 through 6 in Figure 5, which are the only effective moving parts in the rectifier mechanism. The ratios between them are pairwise equal to 1 . These values are equal to: for gears No. 1: $+\theta_{6}+\theta_{5}-\theta_{4}$, No. 2: $+\theta_{6}+\theta_{5}-\theta_{4}$, No. $3:+\theta_{5}+\theta_{4}$, No. $4:+\theta_{5}+\theta_{4}$, No. 5 : $-\theta_{4}$ and No. 6: $+\theta_{4}$. These rotation values of the gears are given based on the practical rotation angle of the robot's main joints, which can rotate joints No. 4, 5, and 6 in the positive direction.

In the next step, considering the calculations in this section which have determined the rotations of gears No. 1, 3 and 5 of Fig 5, and the relationships of motion between the actuators and the internal mobile components in links No. 2, No. 3 and the base of robot, these were already calculated in section 2 and based on Figs. 2, 3 and 4 (equations No. 1 to 4). Because the rotational axes of those gears (gears No. 1, 3 and 5) and the rotational axes of joints No. 2 and 3 are the same and in one direction we can sum the amounts of those rotational angles so we can calculate the kinematic relationship between the mobile components and the actuators for all six joints of the 6-DOF robotic arm discussed in this paper, considering the positive direction of rotation for the actuators shown in Figure 8. This relationship can be expressed as shown below:

$$
\begin{array}{|l}
\hline \begin{array}{l}
\theta_{(2) a}+\theta_{(3) a} \\
\theta_{(2) a}+\theta_{(3) a} \\
\theta_{(2) a}+\theta_{(3) a} \\
\theta_{(2) a}+\theta_{(3) a}=\theta_{(3) m} \\
\theta_{(2) a}=\theta_{(2) m} \\
\theta_{(1) a}=\theta_{(1) m}
\end{array}+\begin{array}{l}
-\theta_{(4) a}+\theta_{(5) a}+\theta_{(6) a} \\
+\theta_{(4) a}+\theta_{(5) a} \\
-\theta_{(4) a}
\end{array} \\
\end{array} \quad \begin{aligned}
& \theta_{(2) a}+\theta_{(3) a}-\theta_{(4) a}+\theta_{(5) a}+\theta_{(6) a}=\theta_{(6) m} \\
& \theta_{(2) a}+\theta_{(3) a}+\theta_{(4) a}+\theta_{(5) a}=\theta_{(5) m} \\
& \theta_{(2) a}+\theta_{(3) a}-\theta_{(4) a}=\theta_{(4) m} \\
& \theta_{(2) a}+\theta_{(3) a}=\theta_{(3) m} \\
& \theta_{(2) a}=\theta_{(2) m} \\
& \theta_{(1) a}=\theta_{(1) m}
\end{aligned}
$$

Conclusions from equation 4 Conclusions from Figs 5 (from base up to joint 3). (5-1) and 6 (from joint 3 up to end effector). (5-2)
Kinematic relations between actuators apace and joints space for complete robot.

(5-3)

* m: rotation related to the motor of joint; a: rotation related to the joint.

In the kinematic simulation of this robot, it's an important fact that we can calculate the related equations for the angular velocities and the angular accelerations between the actuators and the internal mobile parts by calculating the derivative of both sides of Equation (5-3) with respect to time. These equations provide three sets of information that are necessary for kinematic simulation of the robotic arm that we have assessed in this paper.

\section{SIMULATION PLAN}


Our goal in this section is to examine the kinematic simulation procedure of the robotic arm in MATLAB SimMechanics program. Kinematic simulation of a robotic arm means that we only simulated an open-loop control system for the kinematic elements of the robot's movement, which are position, velocity and acceleration of the joints and the robot's end effector, in the Cartesian system and/or in the Rotational system. Indeed, we do not need to check the other two main issues, one of which is the kinetics element of robot motion. This means that we do not consider the lifting torque exerted on the joints and their enforcement. Other issues are the flexibility of the joints and arms and conducting a vibration analysis of the robot. To apply kinematic simulation on the robotic arm, we should do the following steps in order:

[1] Calculate the path generation of desired kinematic elements: a process that has four internal stages, i.e., 1.1. Calculation of the inverse kinematic; 1.2. Calculation of the velocity of joints based on the speed of the end effector and the Jacobian matrix; 1.3. Calculation of the path-generation formulas based on third or fifth degree polynomials. 1.4. Calculation of the desired kinematic elements to simulate robot in joints space based on the path-generation formulas and the simulation time. The methods of calculation in this section are the same as those for other robotic arms. Therefore, to avoid excessive and repetitive information, we did not provide the equations and their solutions here. For more information, see reference No. [19] (Ch. 4, 5 and 7).

[2] Calculating the values of the kinematic elements of the actuators: which are entered to the robot as command values. These amounts will be calculated on the basis of the values that are defined as desired values for the motion of joints from the path generation block and also the relationships between the actuators space and joints (internal motive parts) space that are created because of this motion transmission mechanism (as explained in section 2.2).

[3] Rotational position error: We implemented kinematic simulation of the robot in the rotational space of the joints, so errors in the rotational position of the joints can be defined as follows.

$$
6[19]) \quad E=\theta_{d}-\theta_{o}
$$

* o: output of simulation values, $\mathbf{d}$ : desired values

Figure 10. Shows a Block Diagram of the Open-Loop Control System of the Kinematic Simulation of This Robotic Arm Based on the Above Discussion.

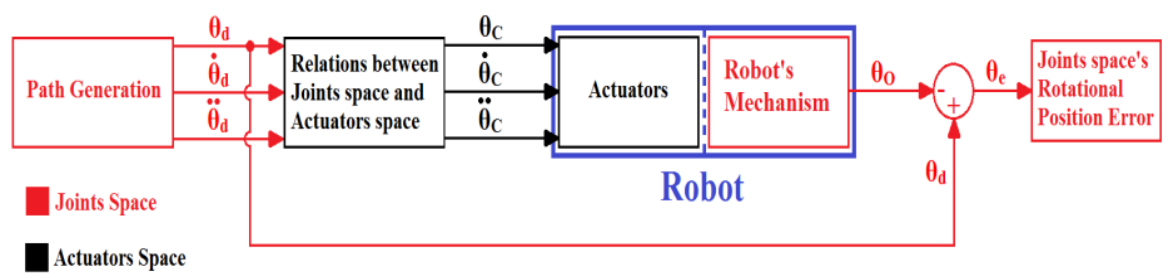

Figure 10. Block Diagram of the Open-Loop Control System of the Kinematic Simulation of Our Robotic Arm.

* d: desired values, $\mathbf{c}$ : command values, o: output of simulation values and e: error values.

The structure of the SimMechanics model for the kinematic simulation of this robotic arm and the open-loop control procedure in that SimMechanics model can be found in Appendix

\subsection{Kinematic properties of simulated robotic arm}

Figure 11 and Table 1 show the frames related to each of the joints, which indicate their kinematic properties along with the Denavit-Hartenberg notation [22] parameters of the robot in this example. (Here, $\mathrm{L} 1=40.5 \mathrm{~mm}, \mathrm{~L} 2=420 \mathrm{~mm}$, and L3 = $442 \mathrm{~mm}$ ). 


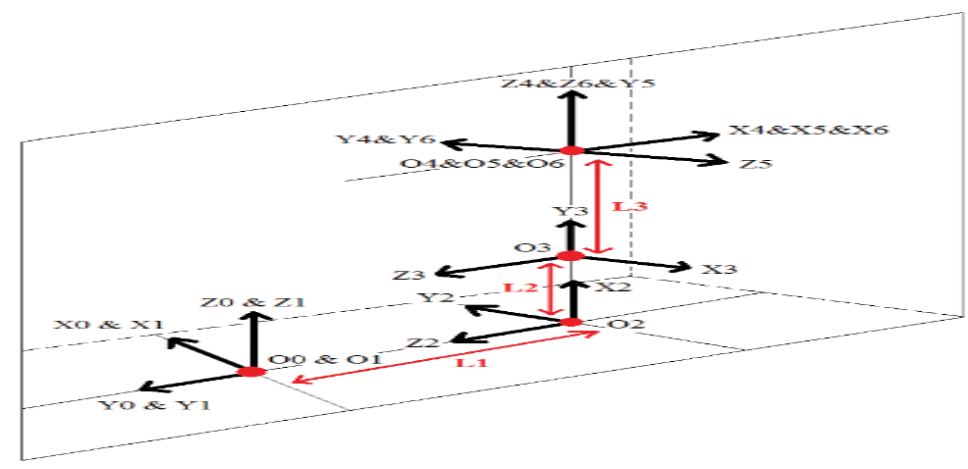

Figure 11. Assignment of Frames Between Each of the Manipulators.

Table 1. Denavit-Hartenberg notation parameters of the robot: $\theta 1$ to $\theta 6$ in this table shows the angle of rotation for the motors of each joint.

\begin{tabular}{ccccc}
\hline $\mathbf{i}$ & $\mathbf{a}_{\mathbf{i}-1}$ & $\boldsymbol{\alpha}_{\mathrm{i}-\mathbf{1}}$ & $\mathbf{d}_{\mathbf{i}}$ & $\boldsymbol{\theta}_{\mathbf{i}}(\mathbf{d e g})$ \\
\hline $\mathbf{1}$ & 0 & $0^{\circ}$ & 0 & $\theta_{1}$ \\
$\mathbf{2}$ & 0 & $-90^{\circ}$ & $-\mathrm{L} 1$ & $\theta_{2}-90^{\circ}$ \\
$\mathbf{3}$ & $\mathrm{L} 2$ & $0^{\circ}$ & 0 & $\theta_{3}-90^{\circ}$ \\
$\mathbf{4}$ & 0 & $-90^{\circ}$ & $\mathrm{L} 3$ & $\theta_{4}+90^{\circ}$ \\
$\mathbf{5}$ & 0 & $+90^{\circ}$ & 0 & $\theta_{5}$ \\
$\mathbf{6}$ & 0 & $-90^{\circ}$ & 0 & $\theta_{6}$ \\
\hline
\end{tabular}

\subsection{Tool Used in the Simulation}

The tool we used for this simulation was a cylinder with a diameter and height of $60 \mathrm{~mm}$. The cylinder was a filled aluminum (AL 2219-T87) block and the density of this metal is $2840 \mathrm{~kg} / \mathrm{m}^{3}$. Regarding the kinematic properties of the tool related to the robot's end effector, we considered the rotation of the frame linked to the tool to be exactly the same as the rotation of the frame of joint No. 6, and there was a distance of $139 \mathrm{~mm}$ between the meeting point of tools to the end effector and the frame of joint No. 6; therefore, the distance between tool's frame and the wrist's frame was $199 \mathrm{~mm}$. According to the explanations about tool from the above, the payload of the robot in this simulation is equal to $\mathbf{4 8 2}$ grs.

\subsection{Designing the Simulation Path}

The motion path we considered as an example to simulate the performance of the robotic arm was composed of four target frames, and Table 2 provides the angles that generated these frames and the angles that generated the origin and destination frames. The motion path for this simulation was designed such that the robot stops for $0.5 \mathrm{~s}$ in the origin frame, moves for $1 \mathrm{~s}$ towards target frame 1 , stops there for $0.5 \mathrm{~s}$, and, then, continues for $1 \mathrm{~s}$ each in its movement towards target frames 2,3 , and 4 . In each of them, we observed a $0.5 \mathrm{~s}$ stop for the end effector. Then, in the return path, the robotic arm came back in the same manner and with the same arrangements, so that the simulation was completed after reaching the destination frame, i.e. the origin frame, and stopping for $0.5 \mathrm{~s}$.

Table 2. Set of Angles Used to Generate Frames for the Origin, Destination and Four Target Points

\begin{tabular}{ccccccc}
\hline $\begin{array}{c}\text { Rotational } \\
\text { Angles }\end{array}$ & Origin & $\mathbf{1}$ & $\mathbf{2}$ & $\mathbf{3}$ & $\mathbf{4}$ & Destination \\
\hline $\mathbf{1 \theta}$ & $0^{\circ}$ & $-64.53^{\circ}$ & $25.47^{\circ}$ & $115.47^{\circ}$ & $205.47^{\circ}$ & $0^{\circ}$ \\
$\mathbf{2 \theta}$ & $0^{\circ}$ & $48.92^{\circ}$ & $48.92^{\circ}$ & $-48.92^{\circ}$ & $-48.92^{\circ}$ & $0^{\circ}$ \\
$\mathbf{3 \theta}$ & $0^{\circ}$ & $35.37^{\circ}$ & $-35.37^{\circ}$ & $35.37^{\circ}$ & $-35.37^{\circ}$ & $0^{\circ}$ \\
$\mathbf{4 \theta}$ & $0^{\circ}$ & $-134.79^{\circ}$ & $-44.79^{\circ}$ & $45.21^{\circ}$ & $135.21^{\circ}$ & $0^{\circ}$ \\
$\mathbf{5 \theta}$ & $0^{\circ}$ & $72.17^{\circ}$ & $36.08^{\circ}$ & $-36.08^{\circ}$ & $-72.17^{\circ}$ & $0^{\circ}$ \\
$\mathbf{6 \theta}$ & $0^{\circ}$ & $-26.14^{\circ}$ & $-52.28^{\circ}$ & $26.14^{\circ}$ & $52.28^{\circ}$ & $0^{\circ}$ \\
\hline
\end{tabular}


Accordingly, the simulation time was $12.5 \mathrm{~s}$, of which $4.5 \mathrm{~s}$ for stopping nine times for $0.5 \mathrm{~s}$ each in the frame locations, and $8 \mathrm{~s}$ were required to move in the eight paths ( $1 \mathrm{~s}$ in each path). The simulation time steps were considered in MATLAB SimMechanics software as variables, and the maximum time interval of these steps taken to be $0.00125 \mathrm{~s}$. The solver for this simulation also was chosen as a trapezoidal solver function and/or ode23t in MATLAB. The lengths of the time steps and type of solver function used had significant effects on the quality of the results and the accuracy of the simulation of the robot's motion. We used the $0.5 \mathrm{~s}$ stop for each of the frames from origin to the destination in order to decrease the errors that occurred in simulate the robot's motion and to provide the robot's structure adequate time to achieve stability.

\section{SIMULATION RESULTS}

In the following and in Fig. 12, the output graphical images of MATLAB SimMechanics software can be observed from the simulation model of the robot's motion as well as the manner of positioning the tool's frame on the goals frames.

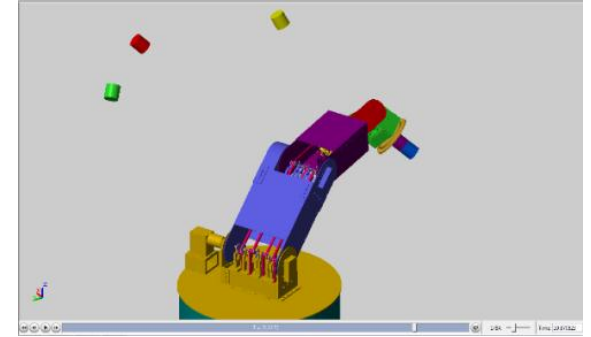

(a) Placement of Frame of Tool on Goal Point Frame No. 1

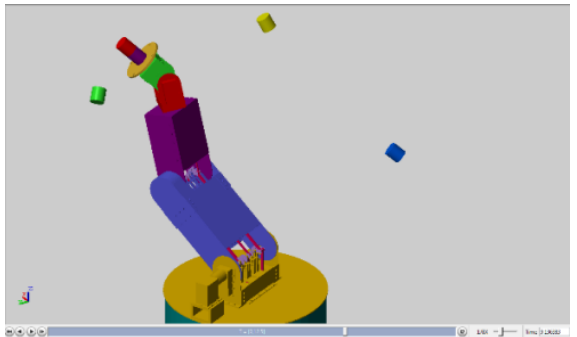

(a) Placement of Frame of Tool on Goal Point Frame No. 2

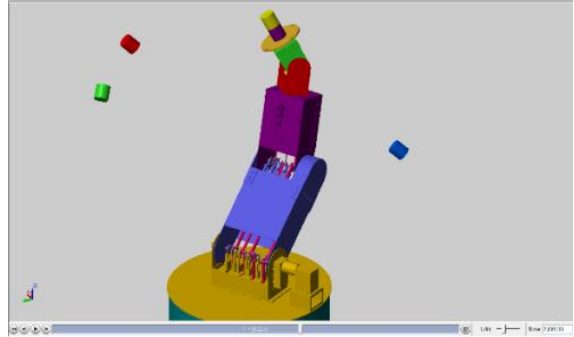

(c) Placement of Frame of Tool on Goal Point Frame No. 3

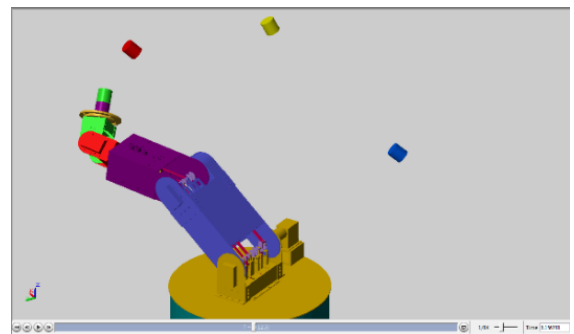

(d) Placement of Frame of Tool on Goal Point Frame No. 4

Figure 12. Images "a" Through "d" Shows Placement of the Tool's Frame of the Robot on Goal Point Frames

Figure 13 shows the 3D drawing of the motion of the robot's end effector (frame connected to joint No. 6 of the robot), both theoretically and output of simulation, where the origin and destination points and the points related to the target frames are named and identified in accordance with the order given in Table 2.

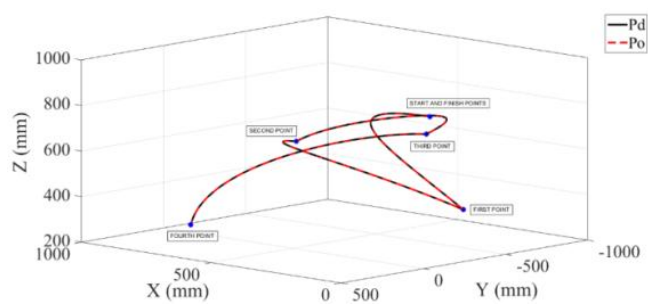

Figure 13. In This Image the Black Continuous Lines Indicate the Desirable Path, While the Dashed Red Lines Show the Output Path in the Simulation for the Position of the Robot's End Effector. 
Figure 14 shows the Theoretical and Output of Simulation Values for the Joints' Space Elements (Rotation Angles of Each of The Joints) During the Robot's Motion Simulation Process.
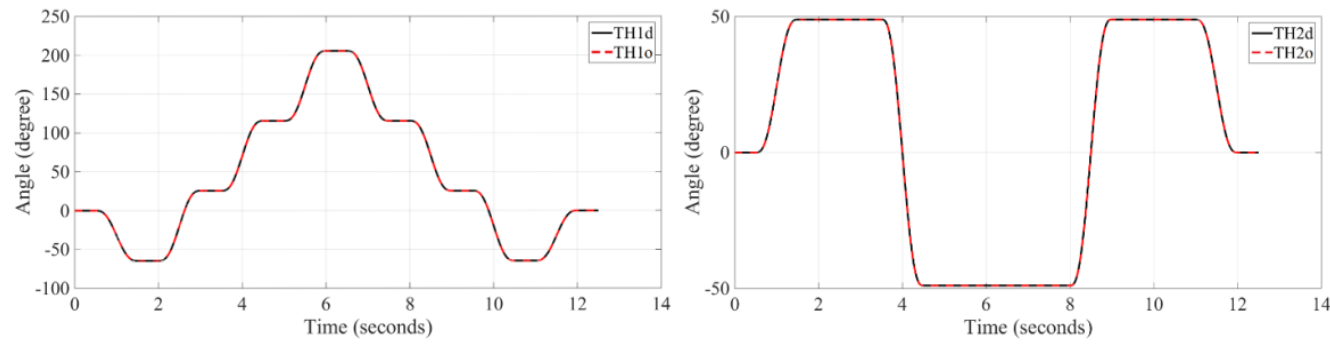

* o: output of simulation values, d: desired values

(a) Left: The trajectories of desired and simulation output values of rotation angle of joint No. 1 Right: The trajectories of desired and simulation output values of rotation angle of joint No. 2
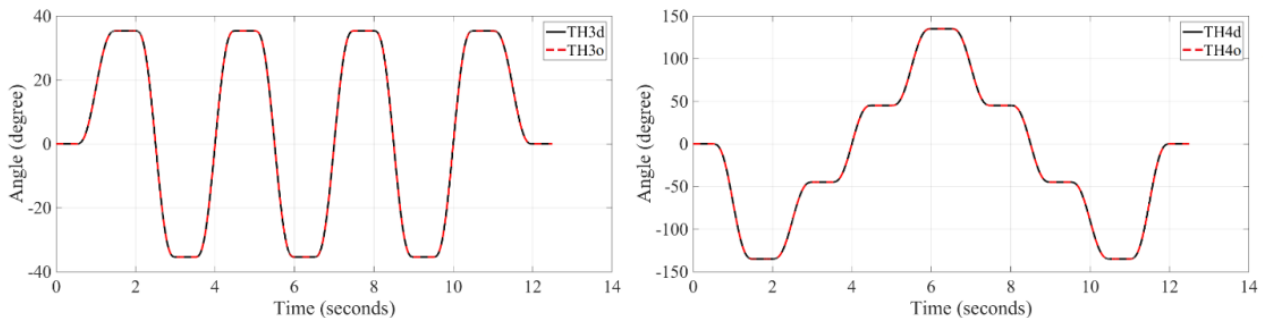

(b) Left: The trajectories of desired and simulation output values of rotation angle of joint No. 3 Right: The trajectories of desired and simulation output values of rotation angle of joint No. 4
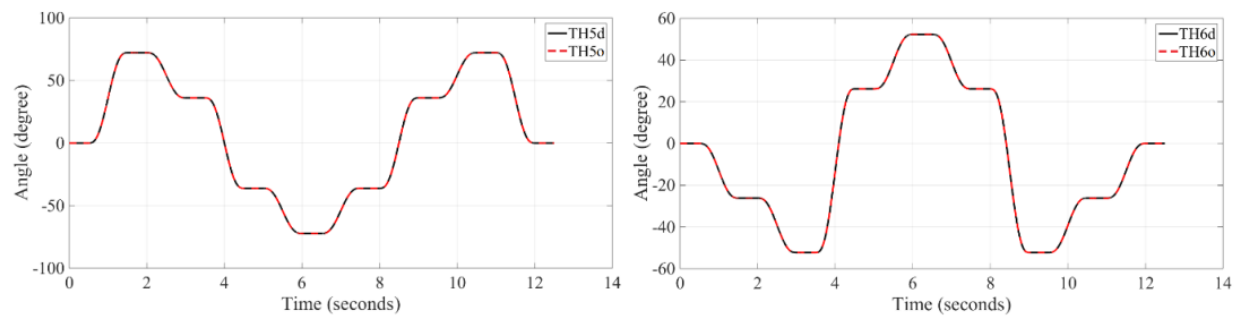

(c) Left: The trajectories of desired and simulation output values of rotation angle of joint No. 5 Right: The trajectories of desired and simulation output values of rotation angle of joint No. 6

Figure 14. Figures "a" through "c" includes the trajectories related to drawing the desired and simulation output rotation angles of joints next to each other.

In terms of degrees, the error we observed between simulation output and desired values relating to the rotation angle of each of the joints and in joints' space are presented in Table 3.

Table 3. Numerical Maximum, Numerical Minimum, And Average Values Of The Errors That Occurred In The Robot's Joints' Rotation Angles.

\begin{tabular}{cccc}
\hline Joints No. & Numerical Max $(\mathbf{d e g})$ & Numerical Min $(\mathbf{d e g})$ & Ave Values $(\mathbf{d e g})$ \\
\hline $\mathbf{1}$ & $2.8422 \mathrm{e}-14$ & $-1.4211 \mathrm{e}-14$ & $1.1207 \mathrm{e}-16$ \\
$\mathbf{2}$ & $7.1054 \mathrm{e}-15$ & $-7.1054 \mathrm{e}-15$ & $6.8950 \mathrm{e}-17$ \\
$\mathbf{3}$ & $1.5989 \mathrm{e}-10$ & $1.6664 \mathrm{e}-11$ & $9.6329 \mathrm{e}-11$ \\
$\mathbf{4}$ & 0.0040 & 0.0040 & 0.0040 \\
$\mathbf{5}$ & -0.0021 & -0.0021 & -0.0021 \\
$\mathbf{6}$ & 0.0047 & 0.0047 & 0.0047 \\
\hline
\end{tabular}




\section{CONCLUSION AND FUTURE WORKS}

In this article, we described our study of the mechanism design and kinematic simulation of a 6DOF robotic arm with rotational joints that move with a linkage motion transmission mechanism and its motors moved to the base of the robot. Our main goal in this research was to lower the joints motors places as much as possible, so that the torque required to be applied to the motors of joints 1,2 , and 3 was decreased to move the robot joints in desirable directions. Regarding the desirable motion implemented by the robot, as it may be seen from the trajectories of theoretical and simulation output values of joints rotational angles as well as the error values in rotation angles for each of the joints, these errors are almost quite little and within as acceptable range, which indicates the fact that those geometrical principles expressed on the manner of performance of this mechanism in section 2 as well as the relations among the actuators space and joints space calculated in section 2.2 are correct. However, regarding the level of performance of this mechanism in comparison to the usual mechanisms, as explained in sections 2 and 2.2, due to using linkage mechanism, there has occurred a dependency among the motions of joints No. 2 thru No. 6, which result in applying specific motion conditions on the joints motors. Depending on the manner of the robot's motion path, these conditions may result in an increase, a decrease, or no considerable change in the level of the rotation angle, velocity, and rotational acceleration imposed to the joints' motors to generate desirable motion. This may, in turn, result in an increase, a decrease, or no considerable change in the level of motion load applied to the robot's motors. Therefore, performance of this motion mechanism in comparison to the ones that are usually used in the robotic arms with 6-DOF rotational joints always will be different, considering the geometrical conditions of motion path, the mechanical specifications of the tools that are used, and the tasks that are assigned. It may be concluded that extensive additional studies are needed to consider the different motion paths the robot should have. This allows us to understand better the level of performance of this mechanism in this kind of robot.

To date, we have not had sufficient time to consider some works that should be conducted to study and solve the problems that persist in this motion mechanism and its practical usage for moving robotic arms, especially the 6-DOF robotic arms with rotational joints. With respect to this concern, however, the following should be noted:

[1] To date, we have had no time to focus on the dynamic control of this mechanism and its use in a robot. This issue is very important, especially as it relates to discussing the design of the robot's mechanical elements, and it definitely is our plan to conduct the required studies in sufficient detail in the future.

[2] In the mechanism that we have presented, it is not easily possible to separate the links in order to determine the resonance frequency of each link. A through assessment of the vibration analysis of this mechanism also is vitally important for the success of the robotic arm. This assessment must take into account the vital importance of accuracy concerning the robot's actions. Thus, we must be able to calculate the position and velocity gains in controlling the parameters that allow us to dynamic control the robot, which involves the appropriate design of the robot's mechanical elements.

[3] The robot's final performance capabilities must be discussed, studied, and calculated to determine the manner and level of the effects associated with backlashes that exist in the mechanical structure of the robot. Such problems can be the result of incorrect workmanship and/or depreciation of parts, so these aspects and their effects on the robot's performance must be evaluated. In addition, this issue has a role in establishing the level of accuracy the robot will be able to attain as well as determining how to modify errors that occur during the robot's movements.

[4] Extensive studies will be required to evaluate the different motion paths, the mechanical attributes of the different tools that are used, and the robot's responses to assigned tasks. In so doing, we will be able to attain a comprehensive understanding of how to achieve the desired levels of performance.

\section{REFERENCES}

[1] R. Aparnathi and V. V. Dwivedi, "The Novel of Six axes Robotic Arm for Industrial Applications", International Journal of Robotics and Automation (IJRA), Vol. 3, No. 3, September 2014, pp. 161 167, ISSN: 2089-4856.

[2] M. D. M. Kutzer, S. M. Segreti, C. Y. Brown, R. H. Taylor, S. C. Mears and M. Armand, "Design of a New CableDriven Manipulator with a Large Open Lumen: Preliminary Applications in the Minimally-Invasive Removal of Osteolysis", 2011 IEEE International Conference on Robotics and Automation, Shanghai International Conference Center, May 9-13, 2011, Shanghai, China.

[3] F. Renda, M. Giorelli, M. Calisti, M. Cianchetti and C. Laschi, "Dynamic Model of a Multibending Soft Robot Arm Driven by Cables", IEEE Transactions on Robotics (Volume: 30, Issue: 5), pp. 1109 - 1122, 09 June 2014, ISSN: 1552-3098. 
[4] Izzeldin. I. M. Abdelaziz, “A Low-Cost Smart Glove for Hand Functions Evaluation”, International Journal of Robotics and Automation (IJRA), Vol. 3, No. 1, March 2014, pp. 39 51, ISSN: 2089-4856.

[5] G. Li, B. Li, J. Sun, W. Zhang, Z. Sun and Q. Chen, "Development of a Directly Self-adaptive Robot Hand with Pulley-belt Mechanism”, International Journal of Precision Engineering and Manufacturing Vol. 14, No. 8, pp. 1361-1368, August 2013.

[6] Peter. V. d. Meulen, inventor; Brooks Automation, Inc., assignee. BATCH SUBSTRATE HANDLING. United States patent, Patent N0.: US 8,950,998 B2. Feb. 10, 2015.

[7] Festo didactic gmbh \& Co, Lab-Volt.ltd, "Robot System Series 5150" ( online product specification, link: http://www1.labvolt.com/publications/datasheets/current2/datasheet_98-5150-0_en_240v_50hz.pdf ).

[8] R. G. Lins, E. W. M. Gomes, M. Corazzim and A. Beaulieu, "Development and Implementation of a Natural Interface to Control an Industrial Hydraulic Robot Arm", Systems Conference (SysCon), 9th Annual IEEE International, pp. 766 - 772, 13-16 April 2015.

[9] [9]. Y. K. Lee and S. J. Lee, “A Bio-Mimetic Robot Arm Actuated by Micro EHA”, 10th International Conference on Ubiquitous Robots and Ambient Intelligence (URAI), Ramada Plaza Jeju Hotel, Jeju, Korea, October 31 November 2, 2013.

[10] KNR Systems, Inc., "Hydraulic robot HYDRA-MP2" (online product specification, link: http://iamknr.gobizkorea.com/catalog/inc/down.jsp?file_path=/att/cat/iamknr/\&file_nm=\%5BBrochure\%5D+H YDRA-MP2.pdf ).

[11] A. Rezoug, B. Tondu, M. Hamerlain, "Experimental Study of Nonsingular Terminal Sliding Mode Controller for Robot Arm Actuated by Pneumatic Artificial Muscles", 19th World Congress The International Federation of Automatic Control Cape Town, South Africa. August 24-29, 2014.

[12] [12]. R. Kang, D. T. Branson, T. Zheng, E. Guglielmino and D. G. Caldwell, "Design, modeling and control of a pneumatically actuated manipulator inspired by biological continuum structures", Bioinspiration \& Biomimetics, Volume 8, Number 3, 2013.

[13] Festo AG \& Co. KG, “Airic's_arm” (online product specification, link: http://www.festo.com/net/SupportPortal/Files/42058/Airics_arm_en.pdf ).

[14] E. G. Christoforou and N. V. Tsekos, "Robotic manipulators with remotely-actuated joints: Implementation using drive-shafts and u-joints", Proceedings of the 2006 IEEE International Conference on Robotics and Automation, Orlando, Florida - May 2006.

[15] N. Hua, Z. Xie, L. Luo, X. Chen, D. J. Tang and H. Zhang, "Design and Analysis in Multiple-Scissor-Linkage Applied to the Robotics Arm", 3rd International Conference on Mechatronics, Robotics and Automation (ICMRA 2015).

[16] S. J. Blumenkranz and D. J. Rosa, inventors; Intuitive Surgical, Inc., assignee. Manipulator positioning linkage for robotic surgery. United States patent, Patent N0.: US 6,246,200 B1. Jun. 12, 2001.

[17] E. Demaine, C. Hoberman and D. Rus.: Mechanical invention through computation: mechanism basics. In: MIT Class 6.S080 (AUS) (2013).

[18] J. P. Merlet, "Parallel Robots, Second edition", INRIA, Sofia-Antipolis, France, Springer Publication, 2006.

[19] Introduction to Robotics, Mechanics and Control, $3^{\text {rd }}$ ed., by John J. Craig, translated by A. Meghdari, F. Mirfakhraei, M. Akrami and E. S. Barjouei, Sharif University of Technology Press, Iran, 2009.

[20] Tabellenbuch Metall, U. Fischer, M. Heinzler and R. Kilgus, translated by A. Valinejad, Tarrah Press, Iran, fall 2012.

[21] MatWeb, "Material Data of Stainless Steel 304," "Material Data of Aluminum 2219-T87," and "Material Data of Titanium Ti-8Al-1Mo-1V," (online specifications, links: http://www.matweb.com/search/datasheet_PDF.aspx?MatGUID=abc4415b0f8b490387e3c922237098da , http://www.matweb.com/search/datasheet_PDF.aspx?MatGUID=86215ca4233a49fdb6b556235fc726b7 and http://www.matweb.com/search/datasheet_PDF.aspx?MatGUID=3a4e0310aa45410fabcc4216982c478c ).

[22] J. Denavit and R. S. Hartenberg, “A Kinematic Notation for Lower-Pair Mechanisms Based on Matrices," Journal of Applied Mechanics, pp. 215-221, June 1955.

\section{Supplementary materials}


We have made 4 captured videos about the operation of our robotic arm in the kinematic simulation process of it which has been simulated in the MATLAB SimMechanics software. They have published along the manuscript of this article and they are named "capture-1" to "capture-4". "capture-1" and "capture-3" show the kinematic simulation of robotic arm in two different views and "capture-2" and "capture-4" show same views of kinematic simulation respectively as shown in the "capture-1" and "capture-3", just with additional show of the coordinate systems frame of each separate part and each goal. To reduce the capture videos size, we have made them directly from the screen of computer while the simulation of the robotic arm was being run and didn't use the capture option of MATLAB SimMechanics software, because the capture files output would have huge size.

\section{Appendix A}

We used from second-generation of MATLAB SimMechanics software for kinematic simulation of this robotic arm. This SimMechanics model has two main units: 1: Controller Unit and 2: Robot's Body Unit.

[1] Controller Unit: the duties of this unit are receiving input data of controller program, doing control process, sending the controller output data to the robot's actuators and at last calculating the error trajectories.

[2] Robot's Body Unit: this unit consists of some blocks which are simulate the structure of robot's motion mechanism and the robot's joints and constraints too. Among these blocks we can mention to: revolute joint blocks, weld joint blocks, bevel gear constraint blocks, body complex (red colored) blocks and etc.

You can find the scheme of this SimMechanics model on below and in Figure A. 1.

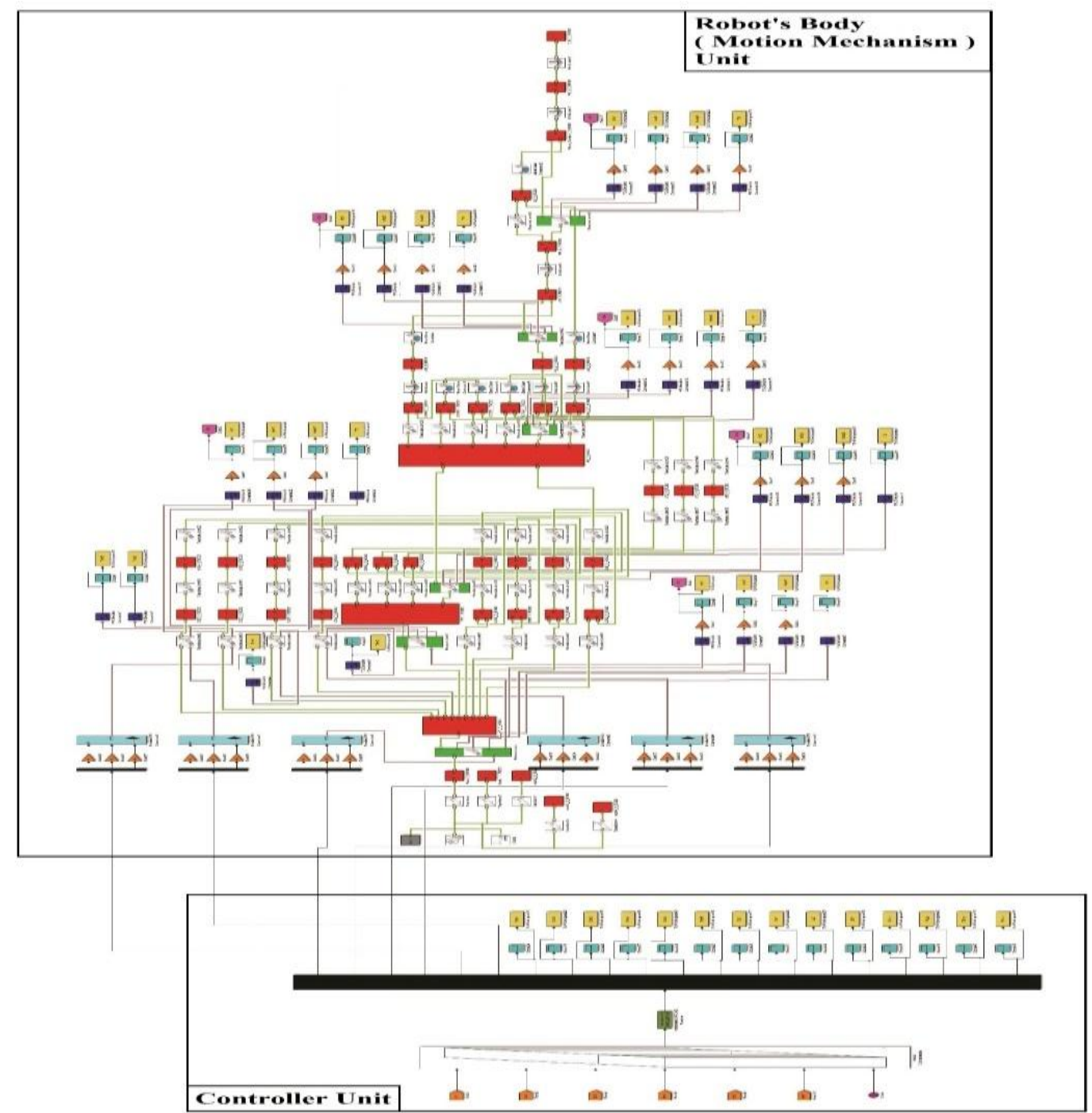

Fig A 1. The scheme of SimMechanics model of kinematic simulation of this robotic arm 